The Twin Continua of Inductive Methods

Paris, J.B. and Vencovska, A.

2015

MIMS EPrint: 2015.67

Manchester Institute for Mathematical Sciences

School of Mathematics

The University of Manchester

\footnotetext{
Reports available from: http://eprints.maths.manchester.ac.uk/

And by contacting: The MIMS Secretary

School of Mathematics

The University of Manchester

Manchester, M13 9PL, UK
}

ISSN 1749-9097 


\title{
J.B.Paris*, A.Vencovská
}

\section{The Twin Continua of Inductive Methods}

\author{
Dedicated to our good friend Jouko
}

\begin{abstract}
After dominating the subject of Inductive Logic for over 50 years Carnap's Continuum of Inductive Methods has in the past decade had its monopoly challenged by a second continuum of inductive methods, the NP-Continuum, which is also based on arguably rational principles. Does this mean there are (at least) two distinct notions of rational or logical probability? We describe the bases and key properties of both continua.
\end{abstract}

Keywords: Uncertain Reasoning, Inductive Logic, Logical Probability, Carnap's Continuum, NP-Continuum

MSC: 03B42,03B48,03A10

*Corresponding Author: J.B.Paris: School of Mathematics, University of Manchester, e-mail: jeff.paris@manchester.ac.uk

\section{Introduction}

A good way to understand the underlying problem that Inductive Logic addresses is as follows: Imagine an agent who inhabits a first order structure $M$ for a language $L$ with relation symbols $R_{1}, R_{2}, \ldots, R_{q}$ and constants $a_{1}, a_{2}, a_{3}, \ldots$ but no function symbols nor equality. Everything in the universe of $M$ is the interpretation of at least one of the $a_{n}$ so we may assume that the set of $a_{n}$ actually is the universe of $M$ with each of the constants interpreted as itself. The agent knows $\mathrm{s} /$ he is in such a structure for $L$ but that is the sum total of the agent's knowledge.

The question now is: Given $\theta \in S L$, where $S L$ is the set of first order sentences of $L$ (and for future reference QFSL is the set of quantifier free sentences of $L$ ), what degree of belief, as subjective probability, should the agent give to $\theta$ being true in $M$ ? Or to take this a step further, what probability function $w: S L \rightarrow[0,1]$ should the agent adopt to reflect his/her beliefs, where by a probability function on $L$ we mean here that $w$ satisfies for $\theta, \phi, \exists x \psi(x) \in S L$,

(P1) $\vDash \theta \Rightarrow w(\theta)=1$

(P2) $\theta \vDash \neg \phi \Rightarrow w(\theta \vee \phi)=w(\theta)+w(\phi)$ 
(P3) $w(\exists x \psi(x))=\lim _{n \rightarrow \infty} w\left(\bigvee_{i=1}^{n} \psi\left(a_{i}\right)\right)$

Although we assume that the agent has no further knowledge of $M$ we should allow that the agent might further consider 'What probability should I give to $\theta$ were I to learn that $\phi$ was true in $M$ ?' The standard convention in the subject is that the agent should in that case take the resulting conditional probability of $\theta$ given $\phi$, to be

$$
w(\theta \mid \phi)=\frac{w(\theta \wedge \phi)}{w(\phi)} .
$$

(A problem seems to arise here when $w(\phi)=0$ but we can satisfactorily side step it by agreeing to the convention that an expression such as $w(\theta \mid \phi)=b$ is actually short for $w(\theta \wedge \phi)=b w(\phi)$, so since $w(\phi)=0$ implies $w(\theta \wedge \phi)=0$, we can in the case $w(\phi)=0$ assign $w(\theta \mid \phi)$ an arbitrary value, for the sake of argument say 1.)

Of course the agent is free to choose any probability function to quantify his/her beliefs. However the name of the game here is that the agent should make a choice which is logical or rational. What that means is that $w$ should be required to satisfy certain principles, principles which we feel it is logical or rational to obey, or more pointedly perhaps which it would be irrational to flout.

For example suppose that $\theta\left(a_{k_{1}}, a_{k_{2}}, \ldots, a_{k_{n}}\right) \in S L$, where the notation is intended to indicate that all the constant symbols appearing in $\theta$ are amongst the (distinct) $a_{k_{1}}, a_{k_{2}}, \ldots, a_{k_{n}}$, and we form $\theta\left(a_{j_{1}}, a_{j_{2}}, \ldots, a_{j_{n}}\right)$ by the obvious simultaneous substitution. Then since the agent has no material reason for thinking that $\theta\left(a_{k_{1}}, a_{k_{2}}, \ldots, a_{k_{n}}\right)$ is any more likely to be true in $M$ than $\theta\left(a_{j_{1}}, a_{j_{2}}, \ldots, a_{j_{n}}\right)$ $\mathrm{s} /$ he should give them each equal probability.

This principle is, unsurprisingly, referred to as Constant Exchangeability, Ex, and it is so widely assumed in this subject that from now on we will assume that all the probability functions we consider satisfy it without further mention. One simplifying consequence of this is that in stating principles we can take the 'arbitrary' $a_{k_{1}}, a_{k_{2}}, \ldots, a_{k_{n}}$ to be $a_{1}, a_{2}, \ldots, a_{n}$ without any loss of generality.

A second principle that we shall assume from the start, both because of its evident rationality and because its presence simplifies the results we wish to state later is:

\section{Regularity, Reg \\ If $\theta \in Q F S L$ is consistent then $w(\theta)>0$.}

The justification here is that given our agent's supposed lack of any specific knowledge about the ambient structure $M$ it would be irrational of him/her to assign probability zero to any consistent quantifier free sentence, in other words 
to assert that as far as s/he was concerned it was impossible for that sentence to hold. ${ }^{1}$

Ex and Reg were principles that W.E.Johnson, [12], and R. Carnap, [1], [2], [3], [4], [5], founders of the subject as a branch of mathematical logic, both employed, Johnson in a period in the late 1920's early 1930's and Carnap, independently, a decade or so later.

The principles Ex and Reg of course give no particular significance to the arity of the relation symbols $R_{1}, R_{2}, \ldots, R_{q}$. However, both Johnson and Carnap concentrated almost exclusively on unary $L$, that is when the $R_{i}$ were all predicates or unary relations. (Indeed it has only been in this millenium that significant advances have been made in Polyadic Inductive Logic, see for example [13].) Like these founding fathers we shall henceforth assume that the $R_{i}$ are all unary.

\section{Carnap's Continuum of Inductive Methods}

A third principle which both Johnson and Carnap proposed became in time known as Johnson's Sufficientness Postulate, JSP after a suggestion by I.J.Good (it started life as Johnson's Sufficiency Postulate but that threatened being confused with the notion of a 'sufficient statistic'). In order to state this principle, and for future use, we first need to introduce a little notation.

Since all our relation symbols are now being taken as unary it follows that everything there is to know about a single constant $a_{i}$ is given by the atom it satisfies, where the atoms of $L$ are those $2^{q}$ formulae $\alpha_{1}(x), \alpha_{2}(x), \ldots, \alpha_{2^{q}}(x)$ of the form

$$
\pm R_{1}(x) \wedge \pm R_{2}(x) \wedge \ldots \wedge \pm R_{q}(x)
$$

$( \pm R(x)$ stands for $R(x)$ or $\neg R(x))$. In turn everything there is to know about $a_{k_{1}}, a_{k_{2}}, \ldots, a_{k_{n}}$ is determined by the state description

$$
\bigwedge_{i=1}^{n} \alpha_{h_{i}}\left(a_{k_{i}}\right)
$$

that they satisfy.

1 The question of whether Regularity should also hold for quantified sentences, in particular consistent sentences of the form $\forall x \theta(x)$ with $\theta$ quantifier free, has sparked a considerable literature, as a lead in see [6, page 87], [10]. 


\section{Johnson's Sufficientness Postulate, JSP}

$$
w\left(\alpha_{j}\left(a_{n+1}\right) \mid \bigwedge_{i=1}^{n} \alpha_{h_{i}}\left(a_{i}\right)\right)
$$

should only depend on $n$ and $m_{j}=\left|\left\{i \mid h_{i}=j\right\}\right|$, i.e. the number of times that $\alpha_{j}(x)$ is instantiated in the state description $\bigwedge_{i=1}^{n} \alpha_{h_{i}}\left(a_{i}\right)$.

One might find an intuitive justification for this postulate by imagining that $M$ was formed by repeated picking (with replacement) copies of atoms $\alpha_{h_{1}}(x), \alpha_{h_{2}}(x), \alpha_{h_{3}}(x), \ldots$ from an urn and requiring that

$$
M=\alpha_{h_{1}}\left(a_{1}\right), \alpha_{h_{2}}\left(a_{2}\right), \alpha_{h_{3}}\left(a_{3}\right), \ldots
$$

With that picture it would seem reasonable that the probability that atom $\alpha_{j}(x)$ would be picked at the $n+1$ 'st stage would only depend on the number of previous picks which had been made and the number of those which had yielded a copy of $\alpha_{j}(x)$.

A reasonably straightforward consequence ${ }^{2}$ of JSP, which we mention because it will figure in what follows, is:

\section{Atom Exchangeability, Ax}

$$
w\left(\bigwedge_{i=1}^{n} \alpha_{h_{i}}\left(a_{i}\right)\right)
$$

depends only on the multiset $\left\{m_{1}, m_{2}, \ldots, m_{2^{q}}\right\}$ where $m_{j}=\left|\left\{i \mid h_{i}=j\right\}\right|$ (as usual).

Equivalently, if $\sigma$ is a permutation of $\left\{1,2, \ldots, 2^{q}\right\}$ then

$$
w\left(\bigwedge_{i=1}^{n} \alpha_{h_{i}}\left(a_{i}\right)\right)=w\left(\bigwedge_{i=1}^{n} \alpha_{\sigma\left(h_{i}\right)}\left(a_{i}\right)\right) .
$$

Interestingly Ax would normally be seen as a principle based on, and indeed rationally justified by, considerations of symmetry whereas JSP is based on irrelevance.

What really makes JSP a major principle in the subject however is the following result which was first proved ${ }^{3}$ by Johnson in [12] and later by Kemeny [11].

2 For details of the proofs of the results stated in this paper collected together in one place see [16] or the forthcoming [18].

3 See [23] for the history of this result and its more general versions. 
Theorem 2.1. Provided $L$ has at least 2 relation symbols (i.e. $q \geq 2$ ) the only probability functions on L satisfying JSP + Reg are the $c_{\lambda}^{L}$ for $0<\lambda \leq \infty$, the so called Carnap's Continuum of Inductive Methods, given by:

$$
c_{\lambda}^{L}\left(\alpha_{j}\left(a_{n+1}\right) \mid \bigwedge_{i=1}^{n} \alpha_{h_{i}}\left(a_{i}\right)\right)=\frac{m_{j}+2^{-q} \lambda}{n+\lambda}
$$

As implied already by the statement of this theorem just the identities afforded by (1) are enough to determine $c_{\lambda}^{L}$ on the whole of $S L$ (though the final extension from QFSL to $S L$ requires a later result due to Gaifman, see [7], or [16, Theorem 7]). We should mention here that if we drop the assumption of Regularity in this theorem then a further solution ${ }^{4}$ appears, $c_{0}^{L}$, defined by

$$
c_{0}^{L}\left(\bigwedge_{i=1}^{n} \alpha_{h_{i}}\left(a_{i}\right)\right)= \begin{cases}2^{-q} & \text { if } h_{1}=h_{2}=\ldots=h_{n}, \\ 0 & \text { otherwise. }\end{cases}
$$

Going to the other extreme, when $\lambda=\infty$, we have that

$$
c_{\infty}^{L}\left(\bigwedge_{i=1}^{n} \alpha_{h_{i}}\left(a_{i}\right)\right)=2^{-n q},
$$

so $c_{\infty}^{L}$ treats all the $R_{j}\left(a_{i}\right)$ as stochastically independent, each with probability $1 / 2$. For $0<\lambda<\infty$ the right hand side of (1) looks like the proportion of $\alpha_{j}$ amongst the $\alpha_{h_{i}}$ moderated by fixed terms in $\lambda$ whose influence tends to zero as $n \rightarrow \infty$. (For a fuller discussion on this and related points see the account by Zabell in [23].)

Theorem 2.1 must be considered one of the most important results in the subject. For it seems to go very far along the road of justifying the idea that considerations of rationality will determine a very tightly restricted choice of probability functions for our agent, indeed a single choice if only $\lambda$ could be fixed unequivocally. Although no generally persuasive argument for any particular value for $\lambda$ has ever emerged, nevertheless we might rightly claim that the continuum of $c_{\lambda}^{L}$ are logical on the grounds that any other rational being will be led inexorably as Johnson and Carnap were to these same choices.

The influence of this position on the later developments of Inductive Logic has been enormous. Most subsequent proposals for logical probability functions to satisfy additional rationality principles not immediately satisfied by the $c_{\lambda}^{L}$ have been based on taking combinations, or adaptations, of the $c_{\lambda}^{L}$. And in justification

4 Sometimes also included in Carnap's Continuum, though not here in this note. 
of this position the $c_{\lambda}^{L}$ do have some attractive features in the context of rational belief.

For example a property that the $c_{\lambda}^{L}$ for $0<\lambda<\infty$ satisfy (directly from (1)) is ${ }^{5}$ :

\section{Reichenbach's Axiom, RA}

If $\alpha_{h_{1}}(x), \alpha_{h_{2}}(x), \alpha_{h_{3}}(x), \ldots$ is an infinite sequence of atoms of $L$ and $m_{j}(n)$ is the number of times that $\alpha_{j}(x)$ appears amongst $\alpha_{h_{1}}(x), \alpha_{h_{2}}(x), \ldots, \alpha_{h_{n}}(x)$ then

$$
\lim _{n \rightarrow \infty}\left(w\left(\alpha_{j}\left(a_{n+1}\right) \mid \bigwedge_{i=1}^{n} \alpha_{h_{i}}\left(a_{i}\right)\right)-\frac{m_{j}(n)}{n}\right)=0
$$

In spirit then, as a recommendation, RA asserts that in the limit the subjective probabilities should converge to frequency. In other words if $34 \%$ of the many people I have ever met have had blue eyes then I should give probability close to $34 / 100$ to the next new person I meet having blue eyes.

A second attractive property is that for fixed $\lambda$ the $c_{\lambda}^{L}$ form a Language Invariant family ${ }^{6}$, meaning that if $L \subseteq L^{\prime}$ (so $S L \subseteq S L^{\prime}$ ) then the restriction of $c_{\lambda}^{L^{\prime}}$ to $S L$ is $c_{\lambda}^{L}$.

This seems clearly justified on the grounds that whilst at any one time our agent might be inhabiting a structure for $L$ with relation symbols $R_{1}, R_{2}, \ldots, R_{q}$ there is no reason to say that these are all the relation symbols there could ever be, and if at a later time the language $L$ was expanded to $L^{\prime}$ then this should not require the agent to revise his/her beliefs about the truth or otherwise of the sentences of the original language $L$.

A final attractive property of the $c_{\lambda}^{L}$ which we mention ${ }^{7}$ is:

\section{The Principle of Instantial Relevance, PIR}

For an atom $\alpha_{j}(x)$ and state description $\Theta\left(a_{1}, a_{2} \ldots, a_{n}\right)$,

$$
w\left(\alpha_{j}\left(a_{n+2}\right) \mid \alpha_{j}\left(a_{n+1}\right) \wedge \Theta\left(a_{1}, a_{2}, \ldots, a_{n}\right)\right) \geq w\left(\alpha_{j}\left(a_{n+1}\right) \mid \Theta\left(a_{1}, a_{2}, \ldots, a_{n}\right)\right)
$$

5 Attributed to H.Reichenbach after a suggestion by H.Putnam, see [4, p120].

6 This notion is usually employed in the polyadic context when we do not restrict our attention to unary predicates so strictly speaking we should be talking here about Unary Language Invariance.

7 This is a slight simplification of a principle of the same name proposed by Carnap, [3]. 
This principle reflects the very heart of what we understand in the real world by 'induction': That the more times I have observed something in the past the more times I should expect to observe it in the future. For example suppose I arrive in a foreign country late at night and waking at 6.00 the next morning I find that it is raining. Shouldn't this increase my belief, or at least not diminish it, that it will also be raining at 6.00 tomorrow morning?

In fact as it turns out the $c_{\lambda}^{L}$ can hardly be said to be particularly distinguished by satisfying PIR since by a result of Gaifman, [8]:

Theorem 2.2. For $\theta\left(a_{n+1}\right), \psi\left(a_{1}, a_{2}, \ldots, a_{n}\right) \in Q F S L$ and $w$ satisfying $E x$,

$$
w\left(\theta\left(a_{n+2}\right) \mid \theta\left(a_{n+1}\right) \wedge \psi\left(a_{1}, a_{2}, \ldots, a_{n}\right)\right) \geq w\left(\theta\left(a_{n+1}\right) \mid \psi\left(a_{1}, a_{2}, \ldots, a_{n}\right)\right)
$$

Whilst this does not particularly promote the primacy of the $c_{\lambda}^{L}$ as such it is an important result because its discovery suggested that rational principles are not only consistent with each other but even to some extent interderivable (and so obliquely that the $c_{\lambda}^{L}$ are their embodiment).

Viewing the above results we might well be led to conclude that in Carnap's Continuum we had uncovered some universal truth about rationality. It all fits together simply and elegantly, isn't that just what we expect of a true theory? Furthermore another characteristic we associate with 'fundamental truths' is that they reoccur as the result of seemingly different approaches (take for example the explication of 'effective process'). That is the case here, there are several principles other than JSP which characterize the $c_{\lambda}^{L}$, see for example [18].

\section{Another Continuum}

Given so many positive features it is scarcely surprising then that for many decades Inductive Logic largely saw Carnap's Continuum as the base camp from which to explore further. In the early 2000's however C. J. Nix and the first author, [14], made a somewhat unexpected discovery.

To explain the background recall the example we gave of observing rain at 6.00 to motivate PIR but now suppose instead that I oversleep and waking at 7.00 note that the streets are wet, though it is not actually raining. Shouldn't that also increase my belief, or at least not diminish it, that it will be raining at 6.00 tomorrow morning? In other words observing a consequence of rain, if not actual rain itself, should still be viewed as supporting evidence for rain tomorrow.

This sentiment might be captured in a principle generalizing Theorem 2.2: 


\section{The Generalized Principle of Instantial Relevance, GPIR}

For $\theta\left(a_{n+2}\right), \phi\left(a_{n+1}\right), \psi\left(a_{1}, a_{2}, \ldots, a_{n}\right) \in Q F S L$, if $\theta(x) \mid=\phi(x)$ then

$$
w\left(\theta\left(a_{n+2}\right) \mid \phi\left(a_{n+1}\right) \wedge \psi\left(a_{1}, a_{2}, \ldots, a_{n}\right)\right) \geq w\left(\theta\left(a_{n+1}\right) \mid \psi\left(a_{1}, a_{2}, \ldots, a_{n}\right)\right)
$$

(As an aside here we mention that this is the same principle if instead we require $\theta(x)$ to be a consequence of $\phi(x)$ rather than the other way round.)

The following theorem, which will appear in [18], is simply a reformulation of results in [14]:

Theorem 3.1. Provided $q \geq 2$ the only probability functions on $L$ satisfying GPIR + $A x+$ Reg are the $w_{L}^{\delta}$ for $-\left(2^{q}-1\right)^{-1}<\delta<1$ given by:

$$
w_{L}^{\delta}\left(\bigwedge_{i=1}^{n} \alpha_{h_{i}}\left(a_{k_{i}}\right)\right)=2^{-q} \sum_{j=1}^{2^{q}} y^{n-m_{j}}(y+\delta)^{m_{j}}
$$

where $y=2^{-q}(1-\delta)$ and (as usual) $m_{j}$ is the number of times that $\alpha_{j}(x)$ is instantiated in the state description $\bigwedge_{i=1}^{n} \alpha_{h_{i}}\left(a_{k_{i}}\right)$.

Interestingly the key power of GPIR here is in allowing additional information to be present in the form of $\psi\left(a_{1}, a_{2}, \ldots, a_{n}\right)$. Without this GPIR holds for all probability functions satisfying $\mathrm{Ax}$, see [18].

So here we have another 'continuum of inductive methods' sometimes referred to as the Nix-Paris (see [22]) or NP-Continuum. As with Carnap's Continuum it is based on rational principles, though the only member that both continua have in common is $c_{\infty}^{L}, w_{L}^{0}$. $^{8}$

For a fixed $\delta$ the $w_{L}^{\delta}$ do not form a Language Invariant family for $-\left(2^{q}-1\right)^{-1}<$ $\delta<0$, this is shown in [15], but they do for $0 \leq \delta<1$ and for this reason it is now common practice (which we henceforth follow) to take the NP-continuum to be the $w_{L}^{\delta}$ with $\delta$ restricted to this range.

In addition to Language Invariance the $w_{L}^{\delta}$ satisfy two other arguably attractive properties. Firstly they satisfy:

\section{The Weak Irrelevance Principle, WIP}

8 In some accounts (1) is extended to the case $\delta=1$, at which point $w_{L}^{1}=c_{0}^{L}$, so meeting again the 'extended Carnap's Continuum' referred to earlier. 
If $\theta, \phi \in Q F S L$ have no relation or constant symbols in common then

$$
w(\theta \mid \phi)=w(\theta)
$$

The intended justification for this principle seems clear: If $\theta$ and $\phi$ come from different languages whyever should knowing the truth of $\phi$ influence one's belief in $\theta$ ?

This property does not provide a characterization of the $w_{L}^{\delta}$, there are other probability functions satisfying Ax + Li which satisfy WIP though they are in a sense all built from the $w_{L}^{\delta}$, see [17]. A property which does however characterize the $w_{L}^{\delta}$ is:

\section{Recovery}

A probability function $w$ on L satisfies Recovery, or is Recoverable, if whenever $\Psi\left(a_{1}, a_{2}, \ldots, a_{n}\right)$ is a state description then there is another state description $\Phi\left(a_{n+1}, a_{n+2}, \ldots, a_{h}\right)$ such that $w(\Phi \wedge \Psi)=0$ and for any quantifier free sentence $\theta\left(a_{h+1}, a_{h+2}, \ldots, a_{h+g}\right)$,

$$
w\left(\theta\left(a_{h+1}, a_{h+2}, \ldots, a_{h+g}\right) \mid \Phi \wedge \Psi\right)=w\left(\theta\left(a_{h+1}, a_{h+2}, \ldots, a_{h+g}\right)\right) .
$$

In other words $w$ is Recoverable if given any 'past history' as a state description $\Psi$ there is a possible 'future' state description $\Phi$ which will take us right back to where we started, at least as far as the quantifier free properties of the currently unobserved constants $a_{h+1}, a_{h+2}, \ldots$ are concerned.

It is debateable whether Recoverability is an obviously desirable property of a rational probability function, though if we think of it as a manifestation of simplicity or at least 'simplifiability', of being able to wipe out the influence of the past, then that could perhaps be argued for. The following result from [20] shows that even a weak form of Recovery (in suitable company at least) actually characterizes the NP-continuum:

Theorem 3.2. Suppose that the probability functions $w_{L}$ satisfy Reg + Ax and form a Language Invariant family. Then $w_{L}$ has the property that for some state description $\Phi\left(a_{1}, a_{2}, \ldots, a_{n}\right)$ with $n>0$, and for all $\theta\left(a_{n+1}, a_{n+2}, \ldots, a_{n+g}\right) \in Q F S L$,

$$
w\left(\theta\left(a_{n+1}, a_{n+2}, \ldots, a_{n+g}\right) \mid \Phi\left(a_{1}, a_{2}, \ldots, a_{n}\right)\right)=w\left(\theta\left(a_{n+1}, a_{n+2}, \ldots, a_{n+g}\right)\right)
$$

just if $w_{L}=w_{L}^{\delta}$ for some $0 \leq \delta<1$. 


\section{Two Rationalities?}

We have seen that we now have two continua, the very well established Carnap's Continuum and the much more recent NP-continuum. Both are 'justified' by satisfying several apparently desirable properties. Indeed it currently seems that most rational properties that we might propose are satisfied by at least one of them.

Comparing these two continua ${ }^{9}$, they have in common that they both satisfy Ax, Reg and Language Invariance. As for their differences the $c_{\lambda}^{L}$ satisfy Johnson's Sufficientness Postulate, which of course the $w_{L}^{\delta}$ do not, and similarly the $c_{\lambda}^{L}$ do not satisfy the Generalized Principle of Instantial Relevance except at $\lambda=\infty$.

A further difference is that apart from the end point $c_{\infty}^{L}$ where the two continua meet the $c_{\lambda}^{L}$ do not satisfy the Weak Irrelevance Property, WIP. As a particular instance of this latter failure one can check that for $0<\lambda<\infty$,

$$
c_{\lambda}^{L}\left(R_{2}\left(a_{3}\right) \wedge R_{2}\left(a_{4}\right) \mid R_{1}\left(a_{1}\right) \wedge R_{1}\left(a_{2}\right)\right)>c_{\lambda}^{L}\left(R_{2}\left(a_{3}\right) \wedge R_{2}\left(a_{4}\right)\right) .
$$

So according to $c_{\lambda}^{L}, R_{1}\left(a_{1}\right) \wedge R_{1}\left(a_{2}\right)$ adds support to $R_{2}\left(a_{3}\right) \wedge R_{2}\left(a_{4}\right)$ despite these two sentences having no relation nor constant symbols in common. However one might argue that this is actually a manifestation of induction which $c_{\lambda}^{L}$ recognizes. Namely the information $R_{1}\left(a_{1}\right) \wedge R_{1}\left(a_{2}\right)$ suggests that the constants look alike, since both $a_{1}$ and $a_{2}$ have been found to satisfy $R_{1}(x)$, and this supports belief in $a_{3}, a_{4}$ also being alike and so both satisfying $R_{2}(x)$. [Actually it turns out that we will always have $\geq$ in (1) for any probability function that is a member of a Language Invariant family of functions satisfying Ax, see [9], so from this point of view the $w_{L}^{\delta}$ might be criticized for not picking up this version of relevance.]

Unlike the $c_{\lambda}^{L}$ (for $\lambda<\infty$ ) the $w_{L}^{\delta}$ do not satisfy Reichenbach's Axiom, RA. To give a specific example of this failure consider the sequence of atoms $\alpha_{h_{i}}(x)$ where $h_{i}=1$ if $i=0 \bmod 3$ and $h_{i}=2$ otherwise. Then with $j=1$ and the notation of the statement of RA, $m_{j}(n) / n \rightarrow 1 / 3$ as $n \rightarrow \infty$. However

$$
w_{L}^{\delta}\left(\alpha_{1}\left(a_{3 n}\right) \mid \bigwedge_{i=1}^{3 n-1} \alpha_{h_{i}}\left(a_{i}\right)\right)=\frac{y(y+\delta)^{2 n}+y^{n+1}(y+\delta)^{n}+\left(2^{q}-2\right) y^{2 n+1}}{(y+\delta)^{2 n}+y^{n+1}(y+\delta)^{n-1}+\left(2^{q}-2\right) y^{2 n}}
$$

which tends to $y$ as $n \rightarrow \infty$, so RA fails. (Of course if $y=1 / 3$ we can just change the frequency of the $\alpha_{1}(x)$.) In defense of the $w_{L}^{\delta}$, there would seem to be no rea-

9 Further comparisons are given in [21]. 
son per se for our agent to suppose that the $m_{j}(n) / n$ would be a convergent sequence. Such a supposition would seem to be based on the rather unwarranted assumption that there was some fixed process, such as picking from an urn with replacement, which was deciding whether or not $a_{n}$ satisfied $\alpha_{1}(x)$. Without this assumption there would seem to be little, or at least less, reason for the agent to slavishly attempt to close in on the 'limit' of the $m_{j}(n) / n$.

Overall though the $c_{\lambda}^{L}$ seem to us to edge it in a straight contest with the $w_{L}^{\delta}$. However that is not the main issue. The main issue is in explaining the alternate concepts of 'rationality' which underpin them.

One might object here that if we accept as our rational probability function the only member that the two continua have in common, i.e. $c_{\infty}^{L}=w_{L}^{0}$, then we get the best of both worlds and there needs be no dilemma. Unfortunately it is rather that we seem get the worst of both worlds. In particular the failure of RA for the $w_{L}^{0}$ is especially dramatic: $w_{L}^{0}$ does not respond at all to the evidence $\bigwedge_{i=1}^{n} \alpha_{h_{i}}\left(a_{i}\right)$ and just gives $\alpha_{j}\left(a_{n+1}\right)$ probability $2^{-q}$ regardless. In other words $w_{L}^{0}$ is entirely devoid of induction and largely in consequence of this few in the area would nowadays wish to promote it as 'the rational choice'. ${ }^{10,11}$

Turning back then to the question of their underlying versions of rationality one might hazard that the difference is this: The principles underlying the $c_{\lambda}^{L}$ require the agent to picture his/her ambient structure $M$ as the result of some regular statistical process. To repeat ourselves, the example of picking balls from an urn in the case of JSP. So the notion of rationality being taken here is of regularity, predictability, pattern. On the other hand the version of rationality being exploited, and subsequently manifest, in the case of the $w_{L}^{\delta}$ might be seen as simplicity or economy. For example the consideration that information should be ignored, or have the potential to be ignored, where possible leads to GPIR, WIP and Recoverability.

10 But see [22] for a spirited counter argument.

11 Another possibility is to extend the continua to $c_{0}^{L}$, $w_{L}^{1}$ where they again agree and make this choice. This has certainly been proposed in its time (and is even inevitable if one pushes symmetry to the limit with purely unary languages, see [19]) but suffers the opposite defect from $c_{\infty}^{L}$ of being too much influenced by the evidence, as shown already in (2). 


\section{Conclusion}

In this paper a number of rationality principles have been proposed each of which is satisfied by, or even characterizes, the probability functions in one of two 'Continua of Inductive Methods', Carnap's classical continuum and the recently explicated NP-continuum. We would suggest that what is significant here is that it implies that there are at least two separate notions of rationality at work and that they are essentially incompatible, or at least their common ground does not provide acceptable rational or logical probability functions to most people's way of thinking.

\section{Bibliography}

[1] Carnap, R., Logical Foundations of Probability, University of Chicago Press, Chicago, Routledge \& Kegan Paul Ltd., 1950.

[2] Carnap, R., The Continuum of Inductive Methods, University of Chicago Press, 1952.

[3] Carnap, R. \& Jeffrey, R.C. eds., Studies in Inductive Logic and Probability, Volume I, University of California Press, 1971.

[4] Carnap, R., A Basic System of Inductive Logic, in Studies in Inductive Logic and Probability, Volume II, ed. R. C. Jeffrey, University of California Press, 1980, pp7-155.

[5] Carnap, R. \& Stegmüller, W., Induktive Logik und Wahrscheinlichkeit, Springer Verlag, Wien, 1959.

[6] Earman, J., Bayes or Bust?, MIT Press, 1992.

[7] Gaifman, H., Concerning Measures on First Order Calculi, Israel Journal of Mathematics, 1964, 2:1-18.

[8] Gaifman, H., Applications of de Finetti's Theorem to Inductive Logic, in Studies in Inductive Logic and Probability, Volume I, eds. R.Carnap \& R.C.Jeffrey, University of California Press, Berkeley and Los Angeles, 1971, pp235-251.

[9] Hill, A. \& Paris, J.B., The Counterpart Principle of Analogical Support by Structural Similarity, to appear in Erkenntnis.

[10] Hintikka, J. \& Niiniluoto, I., An Axiomatic Foundation of the Logic of Inductive Generalization, in Formal Methods in the Methodology of the Empirical Sciences, eds. M.Przelecki, K.Szaniawski \& R.Wójcicki, Synthese Library 103, Kluwer, Dordrecht, 1976, pp57-81.

[11] Kemeny, J.G., Carnap's theory of probability and induction, in The Philosophy of Rudolf Carnap, ed. P.A.Schilpp, La Salle, Illinois, Open Court (1963), pp 711-738.

[12] Johnson, W.E., Probability: The deductive and inductive problems, Mind, 1932, 41(164):409-423.

[13] Landes, J., Paris, J.B. \& Vencovská, A survey of some recent results on Spectrum Exchangeability in Polyadic Inductive Logic, Synthese, 2011, 181(Supplement 1), pp19-47.

[14] Nix, C.J. \& Paris, J.B., A Continuum of Inductive Methods arising from a Generalized Principle of Instantial Relevance, Journal of Philosophical Logic, 2006, 35(1): 83-115. 
[15] Nix, C.J., Nix, C.J., Probabilistic Induction in the Predicate Calculus Doctoral Thesis, Manchester University, Manchester, UK, 2005.

See http://www. maths.manchester.ac.uk/ jeff/

[16] Paris, J.B., Guangzhou Winter School course on Pure Inductive Logic, 2010, available at http://www . maths. manchester.ac.uk/ jeff/lecture-notes/Guangzhou.pdf

[17] Paris, J.B. \& Vencovská, A., A Note on Irrelevance in Inductive Logic, Journal of Philosophical Logic, 2011, 40(3): 357-370

[18] Paris, J.B. \& Vencovská, A., Pure Inductive Logic, to appear in the ASL series Perspectives in Mathematical Logic, Cambridge University Press, 2014.

[19] Paris, J.B. \& Vencovská, Symmetry's End?, Erkenntnis, 2011, 74(1): 53-67.

[20] Paris, J.B. \& Waterhouse, P., Atom Exchangeability and Instantial Relevance, Journal of Philosophical Logic, 2009, 38(3):313-332.

[21] Williamson, J., Inductive Influence, British Journal for the Philosophy of Science, 2007, 58:689-708.

[22] Williamson, J., An Objective Baysian Account of Confirmation, in Explanation, Prediction, and Confirmation. New Trends and Old Ones Reconsidered, Eds. D.Dieks, W.J.Gonzalez, S.Hartmann, T.Uebel \& M.Weber. Volume 2 in the series The Philosophy of Science in a European Perspective, Springer, 2011, pp53-81.

[23] Zabell, S. L., Carnap and the Logic of Induction, in: Handbook of the History and Philosophy of Logic, Volume 10, eds. D.Gabbay, S.Hartmann \& J.Woods, Elsevier, 2011. 